\title{
Briefly Glimpsed People are more Attractive
}

\author{
Don A. Vaughn, ${ }^{1}$ and David M. Eagleman ${ }^{2,}$ \\ ${ }^{1}$ UCLA Semel Institute for Neuroscience and Human Behavior, Los Angeles, CA \\ ${ }^{2}$ Department of Psychiatry and Behavioral Sciences, Stanford University, Stanford, CA \\ Corresponding author: David M. Eagleman, Department of Psychiatry and Behavioral Sciences, Stanford University, Stanford, CA. Tel: +1-7132981825, E-mail: \\ david@eagleman.com
}

Received 2016 February 21; Revised 2016 June 21; Accepted 2016 July 02.

\begin{abstract}
Assessments of attractiveness underlie selection and pursuit of potential mates. Previous research has shown that people are sometimes perceived to be more attractive with a brief glimpse, yet there is no explanation for why this effect might exist. Here, participants rated the attractiveness of males and females photographs, viewed in two conditions: once for $225 \mathrm{~ms}$, and once without time constraints. In the former case, attractiveness judgments were on average higher: briefly glimpsed people were judged to be more attractive. This 'glimpse effect' was most pronounced when males rated photos of females. We discuss several possible explanations for these results, including the speculation that the brain determines attractiveness based on Bayesian risk, in which attractiveness ratings are upwardly biased by the high cost of missing a potential mate.
\end{abstract}

Keywords: Glimpse, Glimpse Effect, Attractiveness, Beauty, Bayesian

\section{Background}

Human faces carry substantial information regarding emotional state and physical well-being $(1,2)$, and so it is not surprising that humans rapidly form initial impressions about someone after a brief exposure (3). One trait which can be extracted fairly accurately in little time is facial attractiveness $(4,5)$.

Interestingly, a recent report by Willis and Todorov shows that subjects who view another person for $100 \mathrm{~ms}$ consider him or her more attractive than if the same person is seen for $500 \mathrm{~ms}$ or $1000 \mathrm{~ms}$ (6). While it is clear that there is less visual information harvested from a brief glimpse, it is not clear, and Willis and Todorov (6) offer no explanation, why the brain might consistently err in one direction in its attractiveness judgements - that is, why stimuli would tend to be interpreted as more attractive instead of less. After all, in many information-processing applications, less information reasonably translates into value judgements of more variability or less confidence: presumably you would not pay more for a car you knew less about.

\section{Objectives}

To understand the reason for the increase in attractiveness judgements in brief exposures, we explore the effect's gender dependency and expand the attractiveness rating scale. We hypothesize that because attractiveness is ultimately related to mate selection, and males are more visu- ally oriented in mate selection $(7,8)$, this temporal effect will be gender dependent.

Additionally, the previous study by Willis and Todorov (6) only used a binary scale (attractive/not attractive), which leaves open the possibility that their effect was driven by a few outliers that changed categories. In other words, did all photographs become a bit more attractive with a brief glimpse (a global shift in the ratings), or did a few outlying photographs simply switch categories? Finally, given our new data, we propose several interpretations and a novel Bayesian framework by which to interpret the results.

\section{Methods}

59 adults (31 female, age $=28.4 \pm 8.4$ years) participated in this study. In total 25 females rated female photographs (FF), 30 females rated male photographs (FM), 24 males rated female photographs (MF), and 21 males rated male photographs (MM). Participants were recruited from the local university community and were compensated for their time.

75 male and 75 female photographs were downloaded from www.hotornot.com. The photos were taken in sequence to preclude selection bias, and were then cropped square and resized to 400 by 400 pixels. To more realistically reproduce the experience of glimpsing a random person, we did not standardize the photo backgrounds or subject hair, clothing, or body posture.

Participants were exposed to all 75 photographs of either the male or female set on a computer screen and rated 
each on a scale from one (least attractive) to ten (most attractive). The photographs were randomly shuffled for each participant and divided into three groups of 25 photographs. Each photograph was displayed in greyscale on a 19" monitor using a Dell PC with MATLAB and the Psychophysics toolbox. At a viewing distance of $59 \mathrm{~cm}$, each photograph subtended $6^{\circ}$ of visual angle.

The test consisted of five blocks. Block 1 used the first group of 25 photographs to acquaint the user with the rating scale and gather average attractiveness ratings. Participants viewed and then rated each photograph with no time constraints ('Long' presentation; Figure 1A).

Figure 1. The Glimpse Effect

A

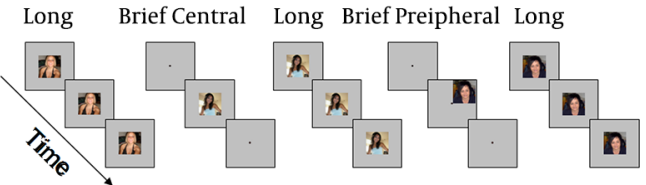

B

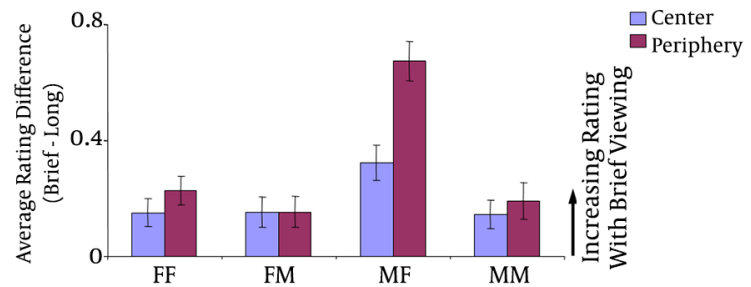

C

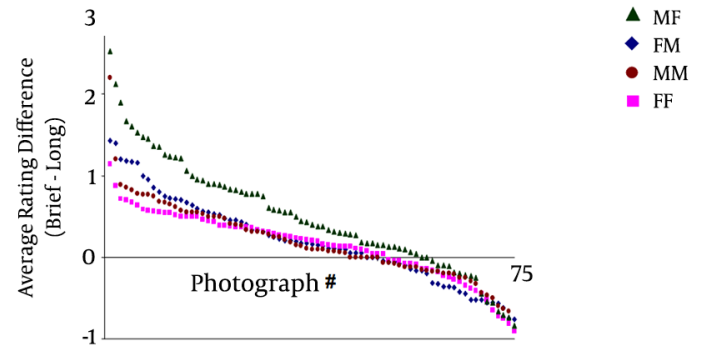

A, The three types of photograph presentations. In the Long presentations, photographs were presented until the participant entered a rating. In the brief central and brief peripheral presentations, photographs were flashed for $225 \mathrm{msec}$, either centrally or in a random location $7^{\circ}$ from fixation dot; B, for each participant and every photo, the attractiveness rating is compared between Brief and Long viewing conditions to quantify the magnitude of the glimpse effect. A positive direction on the y-axis means that brief presentations were judged more attractive than long presentations. The result is significantly more pronounced in males rating female photographs, specifically in the peripheral presentation; $C$, the average difference between Brief and Long ratings for each image (central and peripheral blocks combined). For each category, photographs are sorted on the x-axis in order of decreasing average Brief-Long rating difference. Thus, the first point shows the photograph with the largest glimpse effect in that category.

Block 2 used the second group of 25 photographs. In each trial, participants focused on a red fixation dot in the center of the screen. After a random delay between 500 and 1750 msec, a photograph was flashed in the center of the screen for $225 \mathrm{msec}$ ('Brief Central' presentation, Figure 1A). As before, participants registered attractiveness judgements. In Block 3, participants rated the same photographs as in Block 2, re-presented in a random order, with no time constraints.

To recreate the effect of catching a glimpse "from the corner of one's eye", we presented photos peripherally in Block 4. This Block employed the final group of 25 photographs and was identical to Block 2, with the exception that the photographs were flashed at a random position $7^{\circ}$ from center ('Brief Peripheral' presentation, Figure $1 \mathrm{~A}$ ). The positions of the photographs were randomized to preclude anticipatory saccades. Finally, in Block 5 participants rated the same 25 photographs as in Block 4, re-presented centrally in a random order, with no time constraints.

The order of Brief and Long exposures was not randomized; that is, photographs were always presented briefly before they were re-presented without time constraints. We chose not to randomize the blocks because previous research has shown that exposure to a stimulus improves a person's liking toward it (the 'mere-exposure' effect; (9); similarly, photographs of familiar people are judged to be slightly more attractive than photographs of unknown people $(10,11)$. Thus, in any experiment with repeated presentations, images would likely be rated as more attractive on the second presentation than the first. We structured our experiment to maximally contrast our putative "glimpse effect" against the mere exposure effect. That is, if we were to find that participants report the first, briefly glimpsed exposure to be more attractive, this would demonstrate that the glimpse effect is larger, and in the opposite direction of, the mere exposure effect.

\section{Results}

Confirming previous work by Willis and Todorov (6), observers in all gender categories rated attractiveness significantly higher in both the brief central and brief peripheral presentations than for the Long presentation (Figure 1B; paired t-test; Central: FF P $<0.01$, FM P $<0.01$, MF P $<$ 10 - 6, MM P $<0.01$; Peripheral: FF $\mathrm{P}<10-5$, FM $\mathrm{P}<0.01$, MF $\mathrm{P}<10-20$, MM $\mathrm{P}<0.01)$. We summarize this result as the "glimpse effect". For example, when a male saw a flash of a female in the periphery, he rated her on average 0.673 points higher than when he viewed her in a longer, central presentation (scale from 1 to 10). Although both females and males show the glimpse effect when judging photographs of either gender, the effect is most pronounced in males rating female photographs (Table 1). 
Table 1. The Magnitude and Standard Deviation of the Glimpse Effect in Both Central and Peripheral Presentation for All Four Gender Categories ${ }^{\mathrm{a}}$

\begin{tabular}{lcccc}
\hline & & Central & & Peripheral \\
\cline { 2 - 5 } & Mean & SD & Mean & SD \\
\hline FF & 0.152 & 0.049 & 0.227 & 0.155 \\
FM & 0.153 & 0.052 & 0.673 & 0.054 \\
MF & 0.323 & 0.062 & 0.192 \\
MM & 0.145 & 0.050 & 0.063 \\
\hline
\end{tabular}

${ }^{\mathrm{a}}$ The only difference in the magnitude of glimpse effect between gender groups was in MF; the difference was significant in both the central and peripheral cases (twosample t-test; Central: $\mathrm{P}<0.05$; Peripheral: $\mathrm{P}<10-6$; the p-value given for each category is the least significant of the three comparisons of MF against FM, MF, and $\mathrm{MM})$.

To ensure that our result was not simply caused by a small percentage of the photographs, but instead applied to attractiveness judgements generally, we analyzed the Brief-Long ratings for each photograph individually. The majority of photographs (74\%) generate the glimpse effect (Figure 1C). On average, the effect magnitude for all photographs was significantly greater than zero (two-tailed ttest, $\mathrm{P}<10$ - 6 for female photographs, FF and MF combined; P $<10$ - 3 for male photographs, FM and MM combined). These data verify that the glimpse effect, while not generated by every photograph, holds for most of the photographs in our study.

Is the glimpse effect driven by very high scores becoming low upon longer viewing, or does the effect happen equally across all initial ratings? To address this, we analyzed the change in rating as a function of the initial (brief) rating (Figure 2 ). Results show that essentially all initial ratings are affected equally.

\section{Discussion}

We now consider a few possible explanations for our results. First, poor information derived from a brief glimpse might force the brain's representation of attractiveness to rely on default (prototypical) values. If participants perceived our image set to be less attractive than their default notion of average societal attractiveness, ratings would be higher in the time-constrained presentation, manifesting the glimpse effect. This explanation would suggest that in cases where the glimpse effect occurred, time-constrained attractiveness ratings would be prototypical (towards the middle of the scale). However, as Figure 2 illustrates, we find that the glimpse effect occurs over the entire rating scale, indicating that observers are not relying on default values.

Another possible explanation for our results involves spatial frequencies. Specifically, because high spatial frequencies may require more time to be processed $(12,13)$, fine features that might decrease ratings (e.g., skin blemishes) may be less available in the Brief presentations. This explanation remains a viable possibility, although we would like to suggest a third, non-exclusive possibility as well.

Specifically, we speculatively suggest that the glimpse effect results from the combination of sensory information with the utility of that information (known as a Bayesian risk model, e.g. Geisler and Kersten 2002) (14). As an example, thirsty humans are more likely to perceive ambiguously-transparent stimuli as transparent, revealing a bias toward seeing water (15). Relative to non-thirsty humans, thirsty individuals have an increased utility for water; the value of a hit (finding water) increases relative to that of a false-positive (seeing water when it's not there) thus it behooves a thirsty brain to have a bias towards perceiving transparency. Similarly, the high cost of a miss relative to a false-positive in perceiving sexual intentions might account for males' over-perception of sexual cues from females (16).

This type of Bayesian decision-making, aimed at maximizing expected utility rather than maximum likelihood, might likewise explain the glimpse effect. In contrast to the thirst experiment, which left the stimulus unchanged and varied a participant's utility function, we assumed a constant utility function and varied the stimulus. In our suggested framework, an ideal observer of brief visual input first computes the probability of different attractiveness levels of the stimulus (the posterior probability, Figure $3 \mathrm{~A}$ ). The observer then considers the utility over each outcome and the costs and benefits of mistakes (14, 17-19). To this end, the posterior probability distribution is multiplied by a utility function (Figure 3B), which specifies the benefits of a decision. It is reasonable to assume that a more attractive mate is more valuable, hence we very generally assume a monotonically increasing function. The result of multiplying the posterior probability distribution by the utility function yields the expected utility of each 


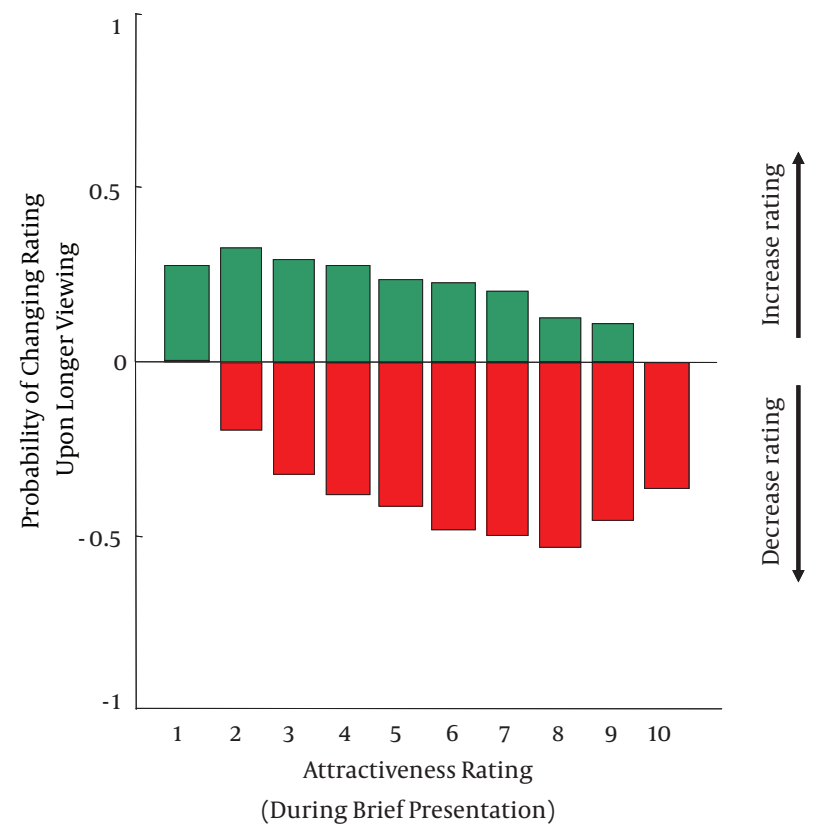

Using the attractiveness rating during the brief presentation as a baseline, the green/red bars indicate the probability that viewers increase/lower their rating in the long viewing. Green bars indicate changes in the opposite direction as the glimpse effect, but these are about one third less likely to occur. Note that the glimpse effect cannot occur for an initial rating of 1 , because the subsequent rating cannot diminish.

choice. An ideal observer will choose the interpretation with the highest expected utility (Figure 3C) instead of simply the maximum probability.

Cast in this framework, the glimpse effect can be explained as an increase in perceived attractiveness in situations of limited information because the cost of an upward bias in the initial assessment of attractiveness (further viewing to confirm initial assessments) is low compared to the cost of a false-negative (failing to identify an attractive potential mate).

The large glimpse effect for males rating females is not surprising in light of the finding that attractiveness judgements depend on the sex of the observer (20), and can presumably be understood in the framework that males place a higher value on physical attractiveness than do females $(21,22)$. Although the glimpse effect was smaller for other gender combinations, note that it was significant in all cases (Figure 2) this result is consistent with the finding that many aspects of attractiveness judgements appear to be independent of gender (23).

We have demonstrated that in males and females, briefly glimpsed photographs of either sex are judged to be more attractive than photographs viewed with no time constraints. While the glimpse effect may be partially due to asymmetrical processing of low and high special frequencies, we additionally suggest that an evolutionary pressure may underlie the glimpse effect: the cost of a miss is high, whereas a false-positive costs only a confirmatory saccade. Optimal decision making requires not only observation about the maximum likelihood of stimuli but utility over possible outcomes - the result of which might explain the existence of many cognitive biases. In this manner, increased utility over greater attraction upwardly biases the percept of stimuli attractiveness. This Bayesian framework of perception is able to explain the significantly greater magnitude of the effect in the case of males rating female photographs as an increase in males' utility function over attractiveness in potential female mates.

To further describe the phenomenon, an important next step is to more precisely characterize the uncertainty in each glimpse. Rather than presenting stimuli with two levels of uncertainty (brief central and brief peripheral), subsequent experiments could vary the temporal and spatial uncertainty across a wider range of values. It may also be of interest to see if the glimpse effect varies with subject age. 
Figure 3. Schematic of the Bayesian Risk Model

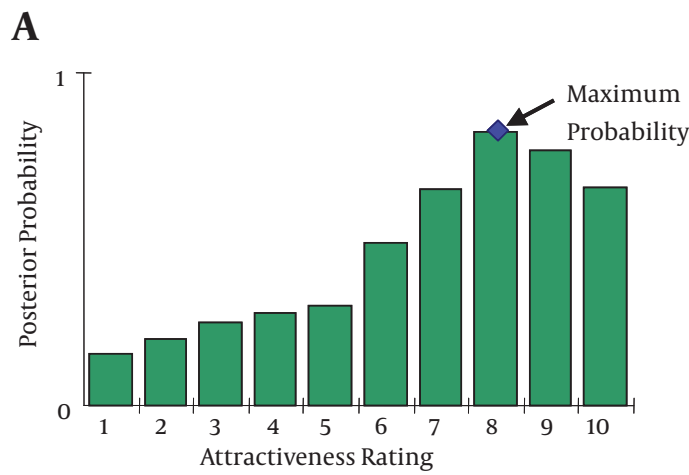

B

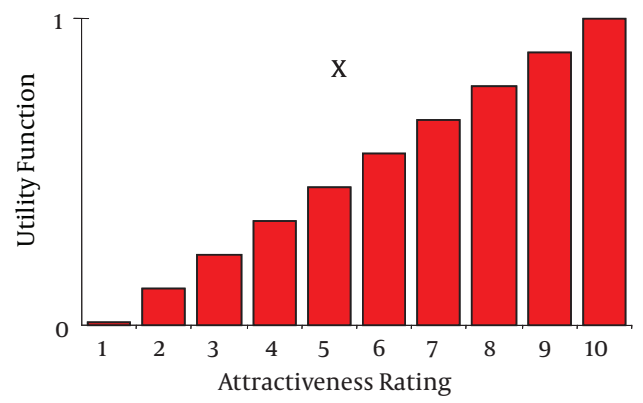

C



$\overline{\text { A, A hypothetical posterior probability distribution; Such a distribution represents }}$ the probability of different stimuli, given sensory input and prior assumptions about the stimuli in the world; B, An observer can incorporate benefits and costs by multiplying the posterior probability distribution by a utility (or cost) function. For illustration, we assume a linear function which makes mate-seeking utility proportional to attractiveness. The shape of the true utility function is unknown. C, The product of the posterior probability and the utility yields expected utility. An ideal observer chooses the decision with the highest expected utility, in this example biasing the judgement toward higher attractiveness.

\section{Acknowledgments}

We thank Alex Holcombe, Keith Kline, Brett Mensh, Chess Stetson and Paul Zak for feedback. Photographs were downloaded from hotornot.com.

\section{Footnote}

Authors' Contribution: Both authors were equally involved in all aspects of the study, from concept and design to manuscript preparation.

\section{References}

1. Hassin R, Trope Y. Facing faces: studies on the cognitive aspects of physiognomy. J Pers Soc Psychol. 2000;78(5):837-52. [PubMed: 10821193].

2. Prkachin KM. The consistency of facial expressions of pain: a comparison across modalities. Pain. 1992;51(3):297-306. [PubMed:1491857].

3. Bar M, Neta M, Linz H. Very first impressions. Emotion. 2006;6(2):26978. doi: 10.1037/1528-3542.6.2.269. [PubMed: 16768559].

4. Locher P, Unger R, Sociedade P, Wahl J. At first glance: Accessibility of the physical attractiveness stereotype. Sex Roles. 1993;28(11-12):729-43. doi: 10.1007/bf00289990.

5. Olson IR, Marshuetz C. Facial attractiveness is appraised in a glance. Emotion. 2005;5(4):498-502. doi: 10.1037/1528-3542.5.4.498. [PubMed: 16366753].

6. Willis J, Todorov A. First impressions: making up your mind after a 100-ms exposure to a face. Psychol Sci. 2006;17(7):592-8. doi: 10.1111/j.1467-9280.2006.01750.x. [PubMed: 16866745].

7. Kenrick DT, Li NP, Butner J. Dynamical evolutionary psychology: individual decision rules and emergent social norms. Psychol Rev. 2003;110(1):3-28. [PubMed: 12529056].

8. Maner JK, Kenrick DT, Becker DV, Delton AW, Hofer B, Wilbur CJ, et al. Sexually selective cognition: beauty captures the mind of the beholder. J Pers Soc Psychol. 2003;85(6):1107-20. doi: 10.1037/00223514.85.6.1107. [PubMed: 14674817].

9. Zajonc RB, Reimer DJ, Hausser D. Imprinting and the development of object preference in chicks by mere repeated exposure.J Comp Physiol Psychol. 1973;83(3):434-40. [PubMed: 4715305].

10. Peskin M, Newell FN. Familiarity breeds attraction: effects of exposure on the attractiveness of typical and distinctive faces. Perception. 2004;33(2):147-57. [PubMed: 15109158].

11. Rhodes G, Halberstadt J, Brajkovich G. Generalization of Mere Exposure Effects to Averaged Composite Faces. Social Cognition. 2001;19(1):57-70. doi: 10.1521/soco.19.1.57.18961.

12. Loftus GR, Harley EM. How different spatial-frequency components contribute to visual information acquisition. J Exp Psychol Hum Percept Perform. 2004;30(1):104-18. doi: 10.1037/0096-1523.30.1.104. [PubMed: 14769071].

13. Vuilleumier P,Armony JL, Driver J, Dolan RJ. Distinct spatial frequency sensitivities for processing faces and emotional expressions. Nat Neurosci. 2003;6(6):624-31. doi:10.1038/nn1057. [PubMed:12740580].

14. Geisler WS, Kersten D. Illusions, perception and Bayes. Nat Neurosci. 2002;5(6):508-10. doi: 10.1038/nn0602-508. [PubMed: 12037517].

15. Changizi MA, Hall WG. Thirst modulates a perception. Perception. 2001;30(12):1489-97. [PubMed: 11817755].

16. Haselton MG. The sexual overperception bias: Evidence of a systematic bias in men from a survey of naturally occurring events.J Res Pers. 2003;37(1):34-47. doi: 10.1016/s0092-6566(02)00529-9.

17. Kersten D, Yuille A. Bayesian models of object perception. Curr Opin Neurobiol. 2003;13(2):150-8. [PubMed: 12744967].

18. Haselton MG, Buss DM. Error management theory: a new perspective on biases in cross-sex mind reading. J Pers Soc Psychol. 2000;78(1):8191. [PubMed: 10653507]

19. Knill DC, Richards W. Perception as Bayesian Inference. UK: Cambridge University Press; 1996.

20. Kranz F, Ishai A. Face perception is modulated by sexual preference. Curr Biol. 2006;16(1):63-8. doi: 10.1016/j.cub.2005.10.070. [PubMed: 16401423]. 
21. Sprecher S, Sullivan Q, Hatfield E. Mate selection preferences: gender differences examined in a national sample. J Pers Soc Psychol. 1994;66(6):1074-80. [PubMed: 8046577].

22. Buss DM, Barnes M. Preferences in human mate selection. J Pers Soc
Psychol. 1986;50(3):559-70. doi: 10.1037/0022-3514.50.3.559.

23. Casey SJ, Mernagh M, Newell FN. Are attractive facial characteristics peculiar to the sex of a face?. Q J Exp Psychol (Hove). 2009;62(5):83343. doi: 10.1080/17470210802592030. [PubMed: 19132630]. 